\title{
The role of the interventional
} cardiologist in selecting antiplatelet agents in acute coronary syndromes: a I 0-question strategy

European Heart Journal: Acute Cardiovascular Care I(2) 170-176

(C) The European Society of Cardiology 2012

Reprints and permission:

sagepub.co.uk/journalsPermissions.nav

DOI: I0.I I77/20488726/2450576

acc.sagepub.com

(SSAGE

\section{Francois Schiele and Nicolas Meneveau}

\begin{abstract}
Antiplatelet agents play a major role in the management of patients with acute coronary syndromes (ACS). In recent years, the most important development has been the advent of new inhibitors of adenosine 5'-diphosphate (ADP) P2Y 12 receptor inhibitors, namely prasugrel and ticagrelor. The arrival of these new drugs on the market, with their specific indications and combinations with aspirin, glycoprotein Ilb/llla inhibitors, and anticoagulants, has rendered the therapeutic arena more complex. Achieving the best combination of all these drugs for each patient requires sound knowledge of the indications of each molecule according to the clinical situation, as well as evaluation of the ischaemic and haemorrhagic risks. In practical terms, the interventional cardiologist holds the key to therapeutic decisions, based on the anatomical information obtained in the cathlab. He/she should be able to recommend an appropriate antiplatelet treatment strategy even before the patient arrives in the cathlab, or alternatively, adapt or modify treatment according to the possibilities for revascularization, and advise on long-term therapy. In this report, we describe, in ten questions, the key elements that the interventional cardiologists should be ready to answer before choosing the appropriate antiplatelet regimen, based on recent guidelines, and covering the whole spectrum of management from pre-hospital, to the cathlab, and after invasive procedures.
\end{abstract}

\section{Keywords}

Acute coronary syndrome, angioplasty, antiplatelet, P2Y I 2 receptor inhibitors, GP IIb/Illa inhibitors

Received: 5 February 20II; accepted: I4 May 2012

\section{Introduction}

Activation of the coagulation cascade and platelet aggregation play a major role in the initiation and spread of arterial thrombus. Therefore, platelets are an ideal therapeutic target in two clinical situations where lesions of the arterial wall can occur, namely coronary angioplasty and acute coronary syndromes (ACS). When performed early enough and with sufficient intensity, platelet inhibition is essential in preventing ischaemic complications and recurrence of thrombotic events. The interventional cardiologist thus occupies a central role in prescribing antiplatelet therapies and is often called upon to advise on the use of antithrombotic drugs before catheterization, or even before having seen the patient at all. In the catheterization laboratory (cathlab), the interventionalist has to continue or complement antiplatelet therapy and adapt therapy to previous choices, all the while taking into account the risk-benefit ratio for the patient. Lastly, he/she also has to advise on the type and duration of antiplatelet treatment for the medium and long terms. This responsibility requires the interventional cardiologist to have a sound knowledge of available antiplatelet agents, the clinical situations for which each molecule has been tested and approved, the indications on the official labelling, and finally, the guidelines for the use of each as issued by professional societies.

Currently, there are eight antiplatelet agents available that act on three main targets, namely the cyclooxygenase pathway with aspirin; the adenosine $5^{\prime}$-diphosphate (ADP) pathway, with four molecules that inhibit $\mathrm{P} 2 \mathrm{Y}_{12}$ receptors

University Hospital Jean Minjoz, Besançon, France.

\section{Corresponding author:}

Francois Schiele, University Hospital Jean Minjoz, Boulevard Fleming, 25000 Besançon, France.

Email: francois.schiele@univ-fcomte.fr 
(ticlopidine, clopidogrel, prasugrel, and ticagrelor); and three inhibitors of glycoprotein (GP) IIb/IIIa receptors, namely tirofiban, eptifibatide, and abciximab. In practical terms, the main difference in treatment regimens resides in the choice of $\mathrm{P}_{2} \mathrm{Y}_{12}$ receptor inhibitors, but this choice then has repercussions on the subsequent use of GP IIb/IIIa inhibitors and anticoagulants.

\section{Three classes of antiplatelet agents used in ACS}

Aspirin is the first step of the therapeutic strategy, with an oral loading dose of 150-300 mg, followed by $75-100 \mathrm{mg}$ per day. The scientific proof of the efficacy of aspirin dates from almost 30 years ago, with a $50 \%$ reduction in ischaemic events as compared to placebo, without any excess of haemorrhagic complications. ${ }^{1}$ Nonetheless, although it may sound simple, the prescription of aspirin is not as clear as it may seem. The efficacy of aspirin has been proven for oral chewed administration, whereas it is often administered intravenously. The intravenous route is fast and effective, but has never been investigated and the most appropriate loading dose of intravenous aspirin remains unclear. The commonly used intravenous aspirin dose of $500 \mathrm{mg}$ achieves systemic drug levels equivalent to $1000 \mathrm{mg}$ given orally. Therefore, the European Society of Cardiology recommends a dose between $80-150 \mathrm{mg}$ of intravenous aspirin. ${ }^{2}$

The second step is another antiplatelet agent: the drug and the appropriate dose depend on the type of infarction and the treatment strategy planned. In the case of ST-elevation myocardial infarction (STEMI), the choice is based on the type of reperfusion strategy chosen, i.e. either thrombolysis or angioplasty. For non-ST-elevation acute coronary syndrome (NSTE-ACS), the choice depends on the level of risk, which takes into account age, haemodynamic conditions, renal function, and troponin elevation. Other criteria also contribute to the decision, such as indication for angioplasty (i.e. coronary anatomy), the level of bleeding risk, and the possible presence of specific contraindications, such as age $\geq 75$ years, body weight $<60 \mathrm{~kg}$, or previous history of stroke.

Among the three most common $\mathrm{P}_{2} \mathrm{Y}_{12}$ receptor inhibitors (ticlopidine is rarely used nowadays because of its adverse side effects, particularly haematological effects), two are thienopyridines that irreversibly inhibit the $\mathrm{P}_{2} \mathrm{Y}_{12}$ receptor, and the third is triazolopyrimidine, a directly acting molecule whose inhibition of the $\mathrm{P} 2 \mathrm{Y}_{12}$ receptor is reversible and is a prodrug.

- Over time, clopidogrel has gradually taken a leading role in numerous situations of arterial disease, both acute and chronic. However, clopidogrel has several critical limitations, namely (1) its relatively weak efficacy, ${ }^{3}(2)$ the high variability in response between individuals, ${ }^{4,5}(3)$ the delayed onset of action of more than 6 hours, ${ }^{6}$ and (4) the fact that it is irreversible.
- By comparison, prasugrel, another irreversible thienopyridine and prodrug, is considerably more powerful, with quicker onset of action and low interindividual variability. ${ }^{3}$ Prasugrel has been tested against clopidogrel in the context of angioplasty at the acute phase of ACS and showed a net clinical benefit through a reduction in thrombotic complications, despite an excess of bleeding complications. ${ }^{7}$ Due to the design of the TRITON study, prasugrel cannot be used upstream of the cathlab, except in case of STEMI, when an invasive strategy by primary angioplasty has already been decided. In this situation, the use of prasugrel is strongly recommended over clopidogrel, since the TRITON study showed a reduction in major adverse events and mortality, without an excess of bleeding events. ${ }^{8}$

- Ticagrelor is a triazolopyrimidine, and like prasugrel, is highly efficacious, with rapid onset of action, and no individual variability. In addition, it presents the novelty of being reversible in 48 hours. ${ }^{9}$ The PLATO study demonstrated a clinical benefit versus clopidogrel, with all clinical situations of ACS, namely STEMI, NSTE-ACS, invasive, conservative and surgical strategies, and even with the possibility of a switch from clopidogrel to ticagrelor. The clinical benefit, particularly a reduction in death (cardiovascular and all cause), was obtained through a reduction in thrombotic events, despite an increase in bleeding complications, except in the situation of coronary artery bypass graft surgery. ${ }^{10}$

The use of GP IIb/IIIa inhibitors upstream of the cathlab has considerably declined in recent years, to the point where their use before the cathlab is now a class III recommendation (i.e. strongly discouraged). ${ }^{11,12}$ The reasons for this 'downgrading' are manifold. Firstly, it has been shown that GP IIb/IIIa inhibitors do not yield any benefit in lowerrisk situations (e.g. ACS without troponin elevation) ${ }^{13}$ or in management strategies comprising medical therapy only (without angioplasty). Furthermore, no superior efficacy was demonstrated with upstream use ${ }^{14,15}$ and studies of GP $\mathrm{IIb} / \mathrm{III}$ inhibitor use with bivaluridin reported an increased bleeding risk without any benefit on ischaemic risk. ${ }^{16,17}$

\section{How to select the adequate regimen?}

In this context, the advice of the interventional cardiologist regarding the appropriate drugs to prescribe in the pre-hospital setting or very early in the management strategy must take into account several clinical scenarios. The decision must be made, at different steps, after discussion with emergency physicians, based on a decision-making algorithm where possible. Several simple questions are useful to choose the appropriate antiplatelet treatment (Figure 1). 


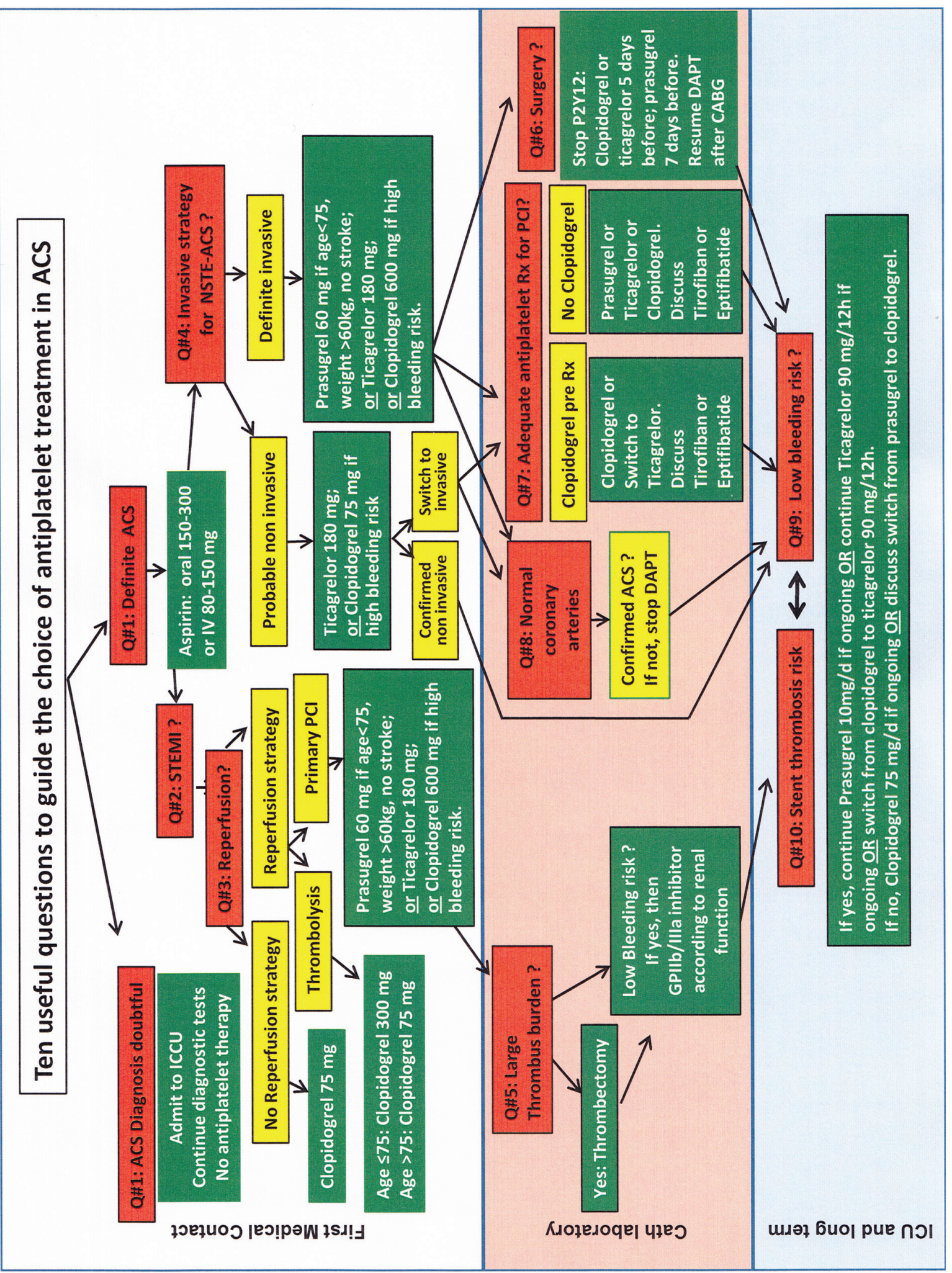

$\stackrel{0}{ \pm}$

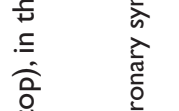

눙

ป

ㅎํㅇ

岂

के

ㅎำ

त

ปे

苞苞

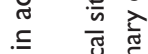

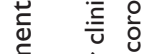

密 弯 ?

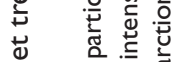

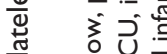

寅

西

ه

응

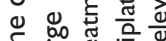

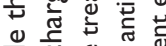

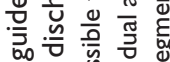

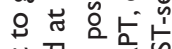

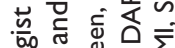

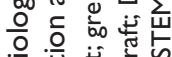

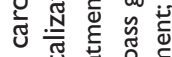

종 끈

을

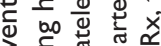

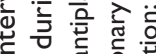

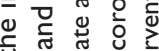

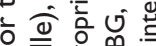

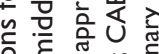

क

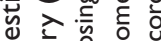

व

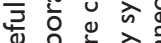

至

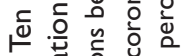




\section{Questions at first medical contact}

The question of antiplatelet therapy arises as soon as ACS is suspected - in other words, long before the patient gets to the cathlab. Early initiation of treatment has a major impact on subsequent angioplasty, by reducing the incidence of a composite of death, myocardial infarction, or urgent revascularization. For this reason, a cardiologist is often consulted at the very first signs of ACS. At this stage in the management, the decision to initiate antiplatelet therapy is usually only based on the clinical probability of ACS and the ECG findings. ${ }^{18}$ Sometimes, these parameters are sufficient to be almost certain of the diagnosis, as is the case with STEMI. However, the clinical conviction is often less certain with NSTE-ACS, where patients often no longer have chest pain by the time they are seen, and the ECG can be normal. ${ }^{19}$

Question I: Is the diagnosis of ACS definite? In case of doubt, it is advisable to refrain from initiating any antiplatelet treatment and to wait for the results of diagnostic tests and risk stratification. Confirmation of the diagnosis of ACS is often based on positive troponin release and this biological result then determines the prescription of antiplatelets, aspirin, and $\mathrm{P} 2 \mathrm{Y}_{12}$ receptor inhibitors. Differential diagnoses for chest pain include situations (such as aortic dissection, pericarditis, gastroduodenal disease) where inappropriate antiplatelet therapy incurs an unnecessary bleeding risk. Confirmation of the diagnosis is of paramount importance: if symptoms are related to the early stages of haemorrhagic syndrome requiring emergency surgery, inappropriate prescription of a loading dose of aspirin and $\mathrm{P} 2 \mathrm{Y}_{12}$ receptor inhibitors can be deleterious. ${ }^{20}$

Question 2: Is it STEMI? The diagnosis of STEMI is confirmed on the basis of symptoms and on the first ECG recording. Double antiplatelet therapy (aspirin and $\mathrm{P} 2 \mathrm{Y}_{12}$ receptors inhibitors) should be initiated in all cases.

Question 3: Is a reperfusion strategy needed and possible? If reperfusion by thrombolysis is planned, only clopidogrel should be used, at a loading dose of $300 \mathrm{mg}$ (or $75 \mathrm{mg}$ if age $>75$ years). ${ }^{21}$ In case of primary angioplasty, prasugrel (loading dose $60 \mathrm{mg}$ ) or ticagrelor (loading dose $180 \mathrm{mg}$ ) should be preferred over clopidogrel $600 \mathrm{mg} .{ }^{11}$ Patients without revascularization should receive $75 \mathrm{mg}$ clopidogrel daily, without any loading dose. ${ }^{22}$

Question 4: In case of NSTE-ACS, can the patient be planned for invasive strategy? Patients with persistent chest pain, with or without ST-segment depression are candidates for invasive strategy: in these cases, ticagrelor and prasugrel are the best options and should be administered as soon as possible, before coronary angiogram (in this situation, prasugrel is indicated for patients in whom angioplasty is planned and who did not already receive clopidogrel ${ }^{12}$ ).
Clopidogrel at a dose of $600 \mathrm{mg}$ is a less favourable option and should be reserved for patients in whom the bleeding risk is considered to be high. ${ }^{12}$

In case of ACS with planned medical strategy, an invasive strategy can be excluded from the outset in certain patients, such as very elderly patients or those with a contraindication to invasive procedures (i.e. patients with a combination of frailty, documented allergy to contrast medium, severely impaired renal function, or difficult arterial access). Ticagrelor (loading dose of $180 \mathrm{mg}$ followed by $90 \mathrm{mg}$ twice daily) is the best option in this case. Clopidogrel (loading dose $300 \mathrm{mg}$ followed by $75 \mathrm{mg}$ maintenance dose) should be reserved for patients with a high bleeding risk. ${ }^{12}$

\section{Questions in the cathlab}

The initial choice of antiplatelet therapy must be revised and adapted according to the findings on the coronary angiogram. Patients with ACS are a very heterogeneous population and the therapeutic options can vary widely according to the coronary anatomy.

Question 5: Is coronary thrombectomy needed? In case of STEMI, coronary occlusion with a relatively large quantity of thrombus is often observed. The emergency nature of the procedure, the presence of thrombus, and the bleeding risk are specific characteristics of STEMI that impact on the choice of antiplatelet agents.

The emergency nature of the procedure leaves little time for $\mathrm{P} 2 \mathrm{Y}_{12}$ inhibitors to achieve their maximum efficacy, and angioplasty is usually performed before the peak efficacy, even when treatment is initiated pre-hospital with a loading dose. This is particularly true for clopidogrel, which achieves maximum efficacy after 6 hours, but is also partially true for prasugrel and ticagrelor. If coronary angiogram reveals a considerable amount of thrombus, the addition of a GP IIb/IIIa inhibitor makes it possible to achieve significant platelet inhibition almost immediately. However, despite this biological advantage, the clinical benefit of GP IIb/IIIa inhibitors in patients pretreated with clopidogrel remains controversial. ${ }^{14,23-26}$ If the decision to add a GP IIb/IIIa inhibitor in the cathlab is made, intracoronary injection of abciximab is possible, but no clinical benefit has been demonstrated.

The TAPAS study validated the use of thrombectomy prior to angioplasty, with a reduction in mortality at 1 year. In this study, thrombectomy was associated with systematic use of GP IIb/IIIa inhibitors (abiciximab) and thus, it is in this specific antiplatelet environment only that a benefit can be expected from thrombectomy. ${ }^{27}$ Indeed, GP IIb/IIIa inhibitors are recommended, even without thrombectomy, in situations of angioplasty with thrombotic lesions, as is the case in ACS, and STEMI in particular. ${ }^{13,24}$

Estimation of the bleeding risk is difficult and usually incomplete in emergency situations and often limited to a 
few major criteria such as age, sex, weight, and previous history of haemorrhagic events. These easily available data nonetheless make it possible to choose appropriately between clopidogrel, prasugrel, and ticagrelor, with the possible addition of a GP IIb/IIIa inhibitor. In patients at high risk of bleeding, apart from using the radial approach, the use of bivalirudin in association with aspirin and clopidogrel offers an alternative to the use of GP IIb/IIIa inhibitors. ${ }^{25}$ Early assessment of renal function is particularly important. Indeed, patients with renal dysfunction are at higher risk of ischaemic and haemorrhagic complications. The presence of impaired renal function will have a major impact on the choice and dose of antithrombotic agents, such as enoxaparin, bivalirudin, eptifibatide, and tirofiban. Enoxaparin and bivalirudin are contraindicated when creatinine clearance is $<30 \mathrm{ml} / \mathrm{min}$; tirofiban and eptifibatide require a dose reduction when creatinine clearance is $<50 \mathrm{ml} / \mathrm{min}$.

Question 6: Is surgical revascularization needed? In case of indication for surgical revascularization, it is recommended that surgery be performed within a few days (provided the clinical situation is amenable to this delay). Since this type of situation presents a high risk of perioperative bleeding, the few days wait provides a window of opportunity to interrupt $\mathrm{P} 2 \mathrm{Y}_{12}$ receptor inhibitor therapy and re-establish platelet function. A class IIa recommendation, with level of evidence $\mathrm{C}$, stipulates that clopidogrel or ticagrelor should be stopped 5 days before surgery, and prasugrel 7 days before, if clinically feasible. ${ }^{11,12}$ In this context, pretreatment with ticagrelor appears to be the best option, since a reduction in mortality has been demonstrated with this molecule versus clopidogrel. ${ }^{28}$

Question 7: Is platelet inhibition adequate before $\mathrm{PCl}$ of the culprit lesion? In NSTE-ACS with a culprit lesion requiring angioplasty, the duration and intensity of prior antiplatelet therapy, as well as the complexity of the lesion and the patient's bleeding risk, should be taken into account when deciding whether to simply maintain antiplatelet therapy or reinforce it.

For $\mathrm{P}_{2} \mathrm{Y}_{12}$ receptor inhibitors, if the patient received 600 $\mathrm{mg}$ of clopidogrel (or $60 \mathrm{mg}$ of prasugrel or $180 \mathrm{mg}$ of ticagrelor) more than 6 hours previously, and the lesion is neither complex nor thrombus-containing, then no modification of treatment is required. ${ }^{29}$

For GP IIb/IIIa inhibitors, conversely, if prior treatment was administered less than 6 hours previously (for clopidogrel, perhaps even less for prasugrel and ticagrelor), or if the lesion contains a considerable amount of thrombus and the patient is at low bleeding risk, then GP IIb/IIIa inhibitors can be considered.

It is not unusual for patients to be sent to the cathlab with a critical coronary lesion without having received any prior treatment by $\mathrm{P} 2 \mathrm{Y}_{12}$ receptor inhibitors. In the absence of any prior treatment, the risk incurred by angioplasty is increased and only the use of GP IIb/IIIa inhibitors makes it possible to proceed with angioplasty without delay. ${ }^{12}$

Question 8: Do the coronary arteries appear normal on angiography? This is not an uncommon finding. In most cases, the initial diagnosis was based on chest pain, more or less typical, associated with troponin elevation. The greater sensitivity of assays (so-called second-generation or ultra-sensitive assays) makes it possible to identify more rapidly and more reliably patients who actually present ACS, but also increases the rate of troponin elevation from non-ischaemic cardiac or non-cardiac causes. In these cases, the diagnosis should be revised and interruption of antiplatelet therapy should be considered. However, spastic angina, a single lesion not seen or not visible on coronary angiogram, or plaque rupture without stenotic atheroma are all potential causes of ACS that require specific diagnostic procedures, as well as continuation of antiplatelet therapy.

There are atheromatous coronary lesions, but no indication for revascularization. In this situation, ticagrelor or clopidogrel should be continued for 1 year in association with aspirin. If GP IIb/IIIa inhibitors have been initiated, they should be stopped.

\section{The interventional cardiologist's opinion after angioplasty}

After the patient has undergone angioplasty and stent implantation, the information acquired by the interventional cardiologist is of major importance for subsequent antiplatelet therapy.

Question 9 and 10: Is the patient at high risk of bleeding or (stent) thrombosis? The two main problems are stent thrombosis and bleeding complications. Among the factors involved in stent thrombosis are early interruption of clopidogrel, as well as inter-individual variability in the response to clopidogrel. The risk of stent thrombosis can be reduced by doubling the dose of clopidogrel $(600 \mathrm{mg}$ loading dose followed by $150 \mathrm{mg}$ for 1 week), ${ }^{29}$ but there are also other possibilities, such as substitution by ticagrelor or prasugrel. Substitution of clopidogrel by prasugrel has not been validated for routine use and is only recommended after stent thrombosis. ${ }^{12}$ Testing for clopidogrel resistance or platelet aggregation is also possible, but generally reserved for specific situations among patients already treated by clopidogrel. However, such tests are not validated for clinical use. ${ }^{12}$ In patients treated with bivalirudin compared with heparin plus a GPIIb/IIIa inhibitor, an excess of stent thrombosis was observed during the first 24 hours, but not when patients were pretreated with unfractionated heparin..$^{25}$ There is no particular recommendation for this situation. ${ }^{11}$ 
Lastly, the type of stent implanted (active or bare metal) should be clearly recorded. Although dual antiplatelet therapy is recommended for 1 year after ACS regardless of the type of stent used, ${ }^{11}$ a range of different events can require early interruption of $\mathrm{P} 2 \mathrm{Y}_{12}$ receptor inhibitors, and when such a decision has to be made, it is vital to know the exact type of stent and date of implantation. This discussion often arises in patients who present an indication for long-term anticoagulant therapy.

\section{Conclusion}

Over the last few years, the use of antiplatelet agents in patients with ACS, and in particular in the cathlab, has become increasingly complex. While aspirin remains current, we have come to understand the limits of clopidogrel, and the advent of new $\mathrm{P} 2 \mathrm{Y}_{12}$ receptor inhibitors have profoundly changed the prescription of antiplatelets, both oral and intravenous. Knowledge of the coronary anatomy and decisions for revascularization heavily influence the choice of antiplatelets, and the interventional cardiologist is a key player to make treatment decisions, not only in the cathlab, but also before and after invasive procedures. Although treatment decisions should be made on a case-by-case basis, the use of decision-making algorithms developed locally in consultation with all physicians involved in the healthcare pathway is possible. These should take into account a limited number of clinical situations, and their appropriate use makes it possible to offer a tailored and personalized antiplatelet regimen to every patient.

\section{Funding}

This research received no specific grant from any funding agency in the public, commercial, or not-for-profit sectors.

\section{Conflict of interest}

FS and NM report receiving grant supports paid to their institution and travel support for scientific meetings, as well as fees for scientific conferences from Astra Zeneca, Boehringer Ingelheim, Daiichi-Sankyo, Lilly, Medtronic, Pfizer; Sanofi, Servier, and Takeda.

\section{References}

1. Theroux P, Ouimet H, McCans J, et al. Aspirin, heparin, or both to treat acute unstable angina. N Engl J Med 1988; 319(17): 1105-1111.

2. Patrono C, Andreotti F, Arnesen H, et al. Antiplatelet agents for the treatment and prevention of atherothrombosis. Eur Heart J 2011; 32(23): 2922-2932.

3. Brandt JT, Payne CD, Wiviott SD, et al. A comparison of prasugrel and clopidogrel loading doses on platelet function: magnitude of platelet inhibition is related to active metabolite formation. Am Heart J 2007; 153(1): 66, e9-e16.

4. Weerakkody GJ, Brandt JT, Payne CD, et al. Clopidogrel poor responders: an objective definition based on Bayesian classification. Platelets 2007; 18(6): 428-435.
5. Collet JP, Hulot JS, Pena A, et al. Cytochrome P450 2C19 polymorphism in young patients treated with clopidogrel after myocardial infarction: a cohort study. Lancet 2009; 373(9660): 309-317.

6. Steinhubl SR, Berger PB, Brennan DM, et al. Optimal timing for the initiation of pre-treatment with $300 \mathrm{mg}$ clopidogrel before percutaneous coronary intervention. J Am Coll Cardiol 2006; 47(5): 939-943.

7. Wiviott SD, Braunwald E, McCabe CH, et al. Prasugrel versus clopidogrel in patients with acute coronary syndromes. $N$ Engl J Med 2007; 357(20): 2001-2015.

8. Montalescot G, Wiviott SD, Braunwald E, et al. Prasugrel compared with clopidogrel in patients undergoing percutaneous coronary intervention for ST-elevation myocardial infarction (TRITON-TIMI 38): double-blind, randomised controlled trial. Lancet 2009; 373(9665): 723-731.

9. Gurbel PA, Bliden KP, Butler K, et al. Randomized doubleblind assessment of the ONSET and OFFSET of the antiplatelet effects of ticagrelor versus clopidogrel in patients with stable coronary artery disease: the ONSET/OFFSET study. Circulation 2009; 120(25): 2577-2585.

10. Wallentin L, Becker RC, Budaj A, et al. Ticagrelor versus clopidogrel in patients with acute coronary syndromes. $N$ Engl J Med 2009; 361(11): 1045-1057.

11. Wijns W, Kolh P, Danchin N, et al. Guidelines on myocardial revascularization. Eur Heart J 2010; 31(20): 2501-2555.

12. Hamm CW, Bassand JP, Agewall S, et al. ESC Guidelines for the management of acute coronary syndromes in patients presenting without persistent ST-segment elevation: The Task Force for the management of acute coronary syndromes (ACS) in patients presenting without persistent ST-segment elevation of the European Society of Cardiology (ESC). Eur Heart J 2011; 32(23): 2999-3054.

13. Kastrati A, Mehilli J, Neumann FJ, et al. Abciximab in patients with acute coronary syndromes undergoing percutaneous coronary intervention after clopidogrel pretreatment: the ISARREACT 2 randomized trial. JAMA 2006; 295(13): 1531-1538.

14. Ellis SG, Tendera M, de Belder MA, et al, Topol EJ. 1-year survival in a randomized trial of facilitated reperfusion: results from the FINESSE (Facilitated Intervention with Enhanced Reperfusion Speed to Stop Events) trial. JACC Cardiovasc Interv 2009; 2(10): 909-916.

15. Giugliano RP, White JA, Bode C, et al. Early versus delayed, provisional eptifibatide in acute coronary syndromes. $N \mathrm{Engl}$ J Med 2009; 360(21): 2176-2190.

16. Stone GW, White HD, Ohman EM, et al. Bivalirudin in patients with acute coronary syndromes undergoing percutaneous coronary intervention: a subgroup analysis from the Acute Catheterization and Urgent Intervention Triage strategy (ACUITY) trial. Lancet 2007; 369(9565): 907-919.

17. Kastrati A, Neumann FJ, Schulz S, et al. Abciximab and heparin versus bivalirudin for non-ST-elevation myocardial infarction. N Engl J Med 2011; 365(21): 1980-1989.

18. Yeh RW, Sidney S, Chandra M, et al. Population trends in the incidence and outcomes of acute myocardial infarction. N Engl J Med 2010; 362(23): 2155-2165.

19. Fox KA, Eagle KA, Gore JM, et al. The Global Registry of Acute Coronary Events, 1999 to 2009 - GRACE. Heart 2010; 96(14): 1095-1101.

20. Schiele F. Double-dose clopidogrel in patients undergoing PCI for ACS. Lancet 2011; 377(9762): 297; author reply 298. 
21. Sabatine MS, Cannon CP, Gibson CM, et al. Effect of clopidogrel pretreatment before percutaneous coronary intervention in patients with ST-elevation myocardial infarction treated with fibrinolytics: the PCI-CLARITY study. JAMA 2005; 294(10): 1224-1232.

22. Chen ZM, Jiang LX, Chen YP, et al. Addition of clopidogrel to aspirin in 45,852 patients with acute myocardial infarction: randomised placebo-controlled trial. Lancet 2005; 366(9497): 1607-1621.

23. Mehilli J, Kastrati A, Schulz S, et al. Abciximab in patients with acute ST-segment-elevation myocardial infarction undergoing primary percutaneous coronary intervention after clopidogrel loading: a randomized double-blind trial. Circulation 2009; 119(14): 1933-1940.

24. Van't Hof AW, Ten Berg J, Heestermans T, et al. Prehospital initiation of tirofiban in patients with ST-elevation myocardial infarction undergoing primary angioplasty (On-TIME 2): a multicentre, double-blind, randomised controlled trial. Lancet 2008; 372(9638): 537-546.

25. Stone GW, Witzenbichler B, Guagliumi G, et al. Bivalirudin during primary PCI in acute myocardial infarction. $N$ Engl $J$ Med 2008; 358(21): 2218-2230.
26. Valgimigli M, Campo G, Percoco G, et al. Comparison of angioplasty with infusion of tirofiban or abciximab and with implantation of sirolimus-eluting or uncoated stents for acute myocardial infarction: the MULTISTRATEGY randomized trial. JAMA 2008; 299(15): 1788-1799.

27. Vlaar PJ, Svilaas T, van der Horst IC, et al. Cardiac death and reinfarction after 1 year in the Thrombus Aspiration during Percutaneous coronary intervention in Acute myocardial infarction Study (TAPAS): a 1-year follow-up study. Lancet 2008; 371(9628): 1915-1920.

28. Held C, Asenblad N, Bassand JP, et al. Ticagrelor versus clopidogrel in patients with acute coronary syndromes undergoing coronary artery bypass surgery: results from the PLATO (Platelet Inhibition and Patient Outcomes) trial. $J$ Am Coll Cardiol 2011; 57(6): 672-684.

29. Mehta SR, Tanguay JF, Eikelboom JW, et al. Doubledose versus standard-dose clopidogrel and high-dose versus low-dose aspirin in individuals undergoing percutaneous coronary intervention for acute coronary syndromes (CURRENT-OASIS 7): a randomised factorial trial. Lancet 2010; 376(9748): 1233-1243. 